\title{
Feasibility of compressive follower load on spine in a simplified dynamic state: A simulation study
}

\author{
Byeong Sam Kim ${ }^{\mathrm{a}, \mathrm{b}}$, Tae-Hong Lim ${ }^{\mathrm{b}}$, Tae Kyu Kwon ${ }^{\mathrm{c}}$ and Kap-Soo Han ${ }^{\mathrm{c}, \mathrm{d}^{*}}$ \\ ${ }^{a}$ Humanetics Innovative Solutions Inc., Plymouth, Michigan, USA \\ ${ }^{b}$ Department of Biomedical Engineering, The University of Iowa, Iowa City, Iowa, USA \\ ${ }^{c}$ Division of Biomedical Engineering, Chonbuk National University, Jeonju, Republic of Korea \\ ${ }^{d}$ Medical School, Chonbuk National University, Jeonju, Republic of Korea
}

\begin{abstract}
This study investigated that the spinal MFs can create compressive follower loads (CFLs) in the lumbar spine in a dynamic state. Three-dimensional optimization and finite element (FE) models of the spinal system were developed and validated using reported experimental data. An optimization analysis was performed to determine the MFs that create CFLs in the lumbar spine in various sagittal postures from $10^{\circ}$ extension to $40^{\circ}$ flexion. Optimization solutions for the MFs, CFLs, and follower load path (FLP) location were feasible for all studied postures. The FE predictions demonstrated that MFs which created CFLs along the base spinal curve connecting the geometrical centers or along a curve in its vicinity (within anterior or posterior shift by $2 \mathrm{~mm}$ ) produced stable deformation of the lumbar spine in the neutral standing and flexed postures, whereas the MFs which created the smallest CFLs resulted in unstable deformation. For extended postures, however, finding CFLs creating MFs that produce stable deformation of the extended spine was not possible. The results of this study support the hypothesis that the spinal muscles may stabilize the spine via the CFL mechanism.
\end{abstract}

Keywords: Compressive follower load, muscle forces, finite element model, lumbar Spine, spine biomechanics

\section{Introduction}

The spine is a long and slender column that supports the upper body. In the 1960s, Nachemson et al. [1] showed that the compressive force acting on the spine may exceed $2600 \mathrm{~N}$, which they estimated by measuring the intradiscal pressure in vivo. However, the ligamentous lumbar spine is known to be unstable when subjected to compressive loads of $88 \mathrm{~N}$ [2]. It is generally accepted that the ligamentous spine is unstable by itself but can be stabilized by the muscle forces (MFs) in vivo.

Experimental studies have shown that, when a compressive force is applied along the spinal curvature (i.e., a follower load), the ligamentous cervical and lumbar spines can withstand compressive loads of up to 250 and $1200 \mathrm{~N}$, respectively, without buckling while maintaining flexibility reasonably well $[3,4]$. The authors of these studies postulated that the follower load corresponds to a physiological

${ }^{*}$ Corresponding author: Kap-Soo Han, Division of Biomedical Engineering, Chonbuk National University, Jeonju, Republic of Korea. Tel.: +82 63250 2541; Fax: +82 63250 2311; E-mail: hanks@jbnu.ac.kr. 
load on the spine in vivo; hence, it has been widely adopted in experimental and analytical studies. Han et al. [5] conducted an analytical study using a three-dimensional (3D) optimization model incorporating the 232 muscles associated with the lumbar spine and demonstrated that numerous combinations of spinal MFs can be used to create a compressive follower load (CFL) in the lumbar spine in various sagittal postures. They also investigated the effect of increasing the external compressive force and flexion moment applied on the lumbar spine and found that the back muscles can create a CFL in the lumbar spine while supporting not only the upper body weight but also a considerable external load. However, this 3D mathematical analysis was performed for static postures under the assumption of a pre-deformed state.

In previous in vitro experimental studies, the action of each individual muscle force has been often ignored, owing to the difficulty of applying loads on a large number of muscles. Therefore, a representative vertical load, often combining with a pure moment, has been used as the loading condition [6-8]. In contrast, in the finite element models, applying muscle forces is relatively easy, but still only a partial number of muscles are implemented, due to the difficulty in applying all unknown muscle forces, and multiple joints have been simplified as deformable one-dimensional (1D) elements in previous studies [9-10]. However, in order to obtain more realistic results, it appears to be important not only to apply individual muscle forces in the finite element model but also to evaluate the spinal system behavior response to applied muscle forces. Therefore, the aim of this study is to investigate the feasibility of the CFL mechanism in a dynamic situation, including deformation of the spinal structure, by applying the individual muscle forces involved in stabilizing the spine during neutral standing, extension, and flexion.

\section{Materials and methods}

A simplified 3D dynamic model using the finite element (FE) method was developed, including several segments as vertebrae, and with spinal muscles attached to the appropriate bony elements. The 3D optimization model developed by Han et al. [5] was adapted to correspond to the loading and boundary condition for this purpose. This model was then used for various finite element analyses using a commercial software package LS-DYNA (Livermore Software Technology, Livermore, CA).

\subsection{FE model description}

The 3D FE model consisted of eight rigid bodies (the trunk (rib cage and T12), lumbar spine (5 vertebrae with ligaments), sacrum, and pelvis) and a total of 232 spinal muscles, as shown in Figure 1(A) and Figure 1(B). The sizes and shapes of the vertebrae (T12 to L5) were determined according to Zhou's CT scan measurements [11]. The lumbar vertebrae between the T12 and the sacrum was located in the appropriate position with the assumed average disc height of $10 \mathrm{~mm}$, in order to provide a lumbar lordosis of $50^{\circ}$ (Cobb's angle measured between the superior endplate of the L1 and the sacrum) [12]. Bone structures were assumed to be rigid and modeled using shell elements with extremely high elastic modulus.

The intervertebral disc with anterior and posterior ligaments was modeled using solid elements, with the nonlinear elastic property determined based on the load-displacement relationships of the lumbar disc, as reported in previous studies [13]. This load-displacement relationship was converted to a stress-strain relationship on the basis of the geometry used in this study for LS-DYNA. The facet joint was modeled using a nonlinear compression-tension spring element to simulate its kinetic characteris- 


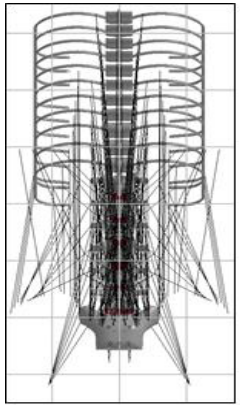

(A)

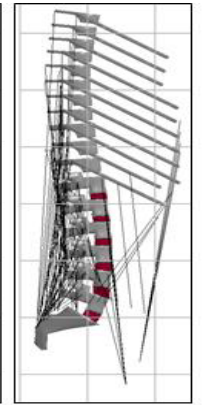

(B)

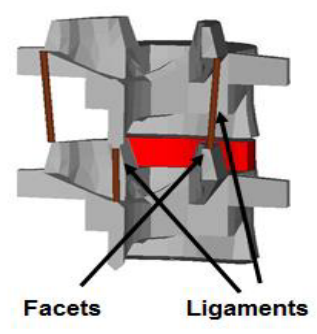

(C)

Fig. 1. 3D FE model of spinal system: (A) posterior view, (B) sagittal view, and (C) one-segment model with springs to simulate the facet joints and ligaments. The lines in (A) and (B) indicate the simulated muscle fascicles.

tics (bony contact and tension in the capsular ligament). The mechanical roles of the remaining ligaments were modeled using one linear tension spring element. The locations of the two springs for facets and one spring for ligaments are shown in Figure 1(C).

A total of 232 muscle fascicles identified in the anatomy literature [14-17] were simulated in the model, including 4 serratus posterior inferior, 14 latissimus dorsi, 6 external oblique, 6 internal oblique, 48 longissimus, 24 iliocostalis, 12 psoas major, 10 quadratus lumborum, 8 rectus abdominis, 6 spinalis thoracis, 40 multifidi, 12 interspinales, 20 intertransversarii, and 22 rotatores. The muscles were modeled using one-dimensional discrete elements, which allow the application of a force of a constant magnitude between the insertion and origin points of each muscle, while allowing the force direction adjustment along the muscle orientation to change during the deformation of the spine. The origin and insertion points of all the muscle fascicles simulated in the previous optimization study [5] were used in this study. Although the pelvis is not shown in Figure 1, the muscle attachment points on the pelvic bone were determined appropriately based upon the CT image of the pelvis. Many of the long muscles (spanning 3 or more motion segments) were forced to pass through several nodes rigidly attached to various vertebrae, in order to simulate their wrapping over the spine during the spinal motions.

\subsection{FE model validation}

The 3D FE model was completed by assigning the material properties to the elements used to simulate the intervertebral discs, facet joints, and ligaments. Using the stress-strain relationship (which was converted from the load-displacement relationship of the intervertebral disc element), the FE model of an isolated motion segment (the vertebral body-disc-vertebral body) not only produced a compressive load-displacement behavior almost the same as that measured in the previous study [13] (Figure 2) but also replicated the range of motion (ROM) for each level that was similar to that of previous studies [18-22] (Figure 3).

To demonstrate a reliable FE simulation of the in vivo lumbar spine with a substantial precompressive load, the disc elements were replaced under the load-displacement relationship by shifting the initial displacement to correspond to a physiological compressive load of $800 \mathrm{~N}[21,22]$ in a neutral standing posture, as shown in Figure 2(A). Although the load-displacement curve in blue represents the compression and flexion-extension mechanical behavior of the disc in vitro (no preload condition), the red curve was used in the current FE analysis to consider the pre-strain of the disc under physiological preloading (in vivo). Then, the segmental and L1 ROMs of this FE model in re- 


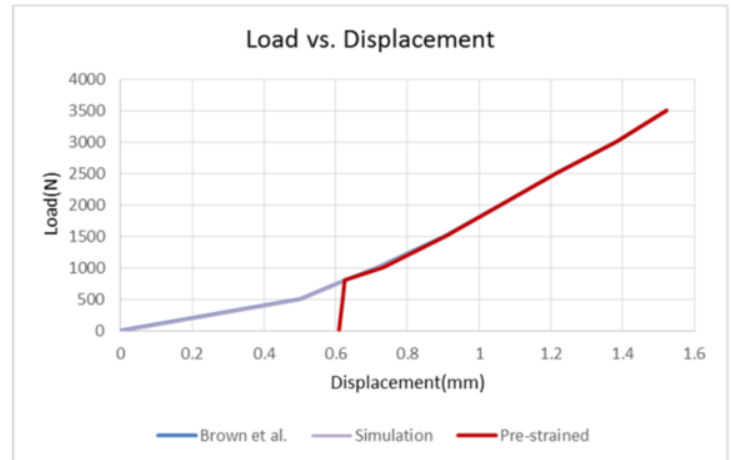

(A)

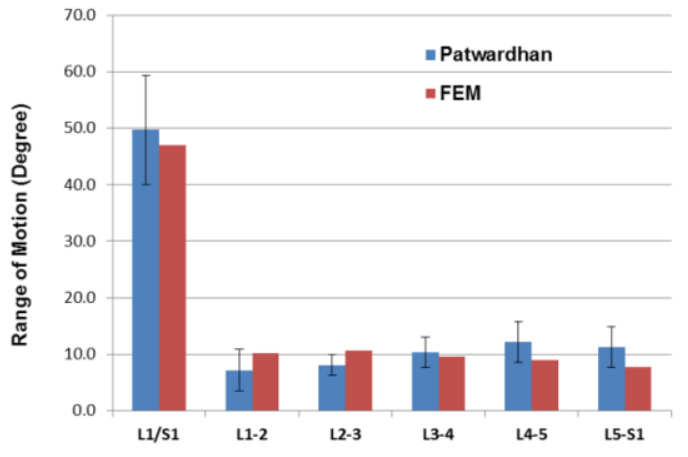

(B)

Fig. 2. (A) Nonlinear load-displacement relationship of disc element used in FE analyses. (B) In vitro experiment and FE simulation of segmental flexion-extension ROM without preloading.

sponse to the moments ( $6 \mathrm{~N} \mathrm{~m}$ in extension and $8 \mathrm{~N} \mathrm{~m}$ in flexion) applied at the L1 vertebra were predicted and compared with those measured in Renner et al.'s [23] tests of the lumbar spine, under the $800 \mathrm{~N}$ compressive pre-load along the spinal curve, as shown in Figure 2(B). This was done in order to validate that the load-displacement behavior of this FE model functions as a realistic model of the lumbar spine under a physiological compressive load. The mean flexion-extension ROM of L1 with regard to S1 as predicted by the FEM $\left(47.1^{\circ}\right)$ demonstrated a good agreement with the experimental results from a previous study conducted by Patwardhan et.al $\left(49.7^{\circ} \pm 9.7^{\circ}\right)$.

\subsection{Optimization analysis}

Following the validation, flexion and extension moments were applied to the L1 vertebra in the FE models in order to obtain the geometries corresponding to the spinal system in various sagittal postures (L1-Sa ROM of $10^{\circ}$ extension, $5^{\circ}$ extension, neutral, $10^{\circ}$ flexion, $20^{\circ}$ flexion, and $40^{\circ}$ flexion). These geometries were then used to develop the optimization models required to predict the MFs creating the CFLs in the lumbar spine in the abovementioned sagittal postures, using the optimization method used in the previous study [5]. Briefly, the previous optimization problem was formulated to determine the MFs that can minimize the internal joint loads (CFLs and moments) while simultaneously satisfying the static equilibrium of forces and moments at all lumbar levels in the thoracolumbar spine in a quiet standing posture.

The mathematical formulation to find the optimal condition of the lumbar spine is

$$
\min (f)=\sum_{l=1}^{6}\left\|F_{l}^{j t}\right\|+\sum_{l=1}^{6}\left\|M_{l}^{j t}\right\|
$$

Subject to

$$
\begin{aligned}
& \sum_{k=1}^{n} \vec{F}_{k, l}^{m}+\sum_{k=1}^{n \prime} \vec{F}_{k, l}^{e x t}+\vec{F}_{l}^{j t}+\vec{F}_{l+1}^{j t}=0(l=1, \ldots, 6) \\
& \vec{F}_{l+1}^{j t} / /\left(\vec{r}_{l+1}^{j t}-\vec{r}_{l}^{j t}\right)(l=1, \ldots, 6)
\end{aligned}
$$




$$
\begin{aligned}
& -30 \leq \rho_{l} \leq 15(l=1, \ldots, 6) \\
& 0 \leq\left\|\vec{F}_{k}^{m}\right\| \leq F_{\max }^{m}(k=1, \ldots, 232)
\end{aligned}
$$

where $\mathrm{F}, \mathrm{M}$, and $\mathrm{r}$ indicate the force, moment and vector to the geometric center (GC) of the vertebral body, respectively, and the superscripts $\mathrm{m}$, ext, and jt represent the muscle, external component (upper body mass or external loads), and joint, respectively. A double subscript divided by a comma indicates the number of components attached to a certain level of vertebra. The location of the curve along which the CFLs followed, or the follower load path (FLP), was described in terms of $\rho$, which indicates the parallel shift of the base spinal curve (established by connecting the GCs of the vertebral bodies) in millimeters either anteriorly $(+\rho)$ or posteriorly $(-\rho)$. For all modeling cases, the maximum MF capacity (MFC), or the maximum contraction force per physiological cross-sectional area, was assumed to be $F_{\max }^{m}<45 \mathrm{~N} / \mathrm{cm}^{2}$ for all muscles. The range of $\rho$ was limited to $-30 \mathrm{~mm}$ to $15 \mathrm{~mm}$ to prevent the FLP forming out of the spinal column from the facet joint to the anterior cortex of the vertebral body. Numerous combinations of MFs creating CFLs along various FLPs were obtained from the optimization models using a generalized gradient method built with the commercial software LINGO (LINDO Systems, Inc., Chicago, IL, USA), as in the previous study

\subsection{FE analysis}

The following loading and boundary conditions were assumed in all cases simulating the quiet standing posture and flexed postures:

1) The upper body weight of $350 \mathrm{~N} \mathrm{[5]} \mathrm{was} \mathrm{assumed} \mathrm{to} \mathrm{be} \mathrm{located} \mathrm{at} \mathrm{the} \mathrm{trunk} \mathrm{center} \mathrm{of} \mathrm{gravity}$ (CG), according to the literature [5], in such a way that the weight produces a flexion moment of $3.5 \mathrm{~N}$ $\mathrm{m}$ about the geometrical center of the T12 vertebra body when the spine is in a neutral (upright standing) posture.

2) The MFs calculated from the optimization models for various cases were added to the FE model as case-specific loading conditions.

3) The sacrum and pelvis were considered fixed, whereas the trunk and all the lumbar vertebrae were allowed to move freely in space.

Out of the numerous FE predictions (stress-strain distributions, linear and angular displacements, rigid bodies, etc.), the displacement of the trunk CG was used to estimate the stability of the lumbar spine because it indicates the sway of the trunk in a static posture. The spinal deformation resulting in the trunk CG displacement smaller than $10 \mathrm{~mm}$ was considered as stable deformation and vice versa [5].

\section{Results}

The ROMs predicted from the sacrolumbar FE model in response to the flexion $(8 \mathrm{~N} \mathrm{~m})$ and extension $(6 \mathrm{~N} \mathrm{~m})$ moments showed excellent agreement with the ROMs measured in the experiment [22] under the compressive follower load of $800 \mathrm{~N}$, as shown in Figure 3(A), not only in the L1-Sa ROM but also in all segmental ROMs. The L1-S1 flexion-extension predicted by FEM with a follower load of $800 \mathrm{~N}\left(41.8^{\circ}\right)$ was in good agreement with the results in vitro $\left(39.0^{\circ} \pm 7.6^{\circ}\right)$; the ROM of the stiffened disc was $33.7^{\circ}$ and also fell within one standard deviation. Such a good agreement indicates that the FE model using stiffened disc properties represents the in vivo (or pre-loaded) lumbar spine beha- 


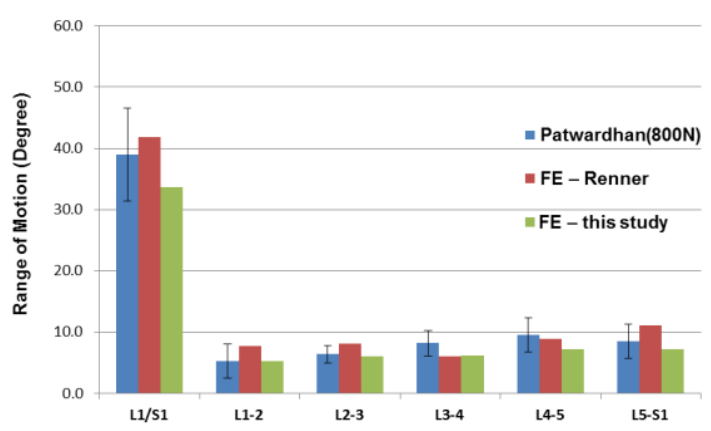

(A)

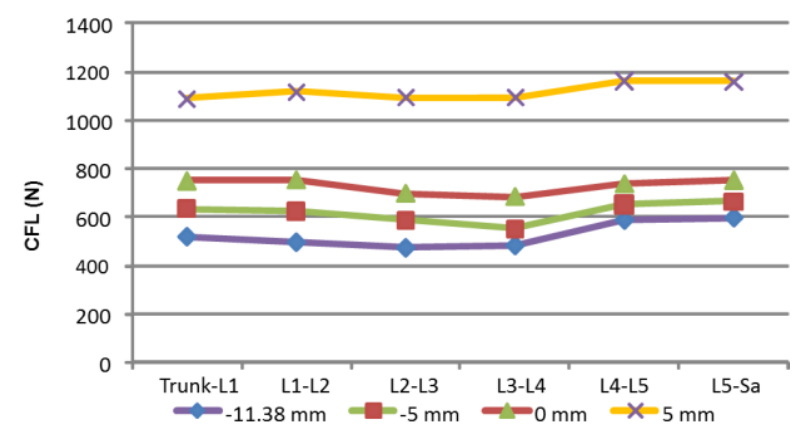

(B)

Fig. 3. (A) In vitro experiment and FE simulation of total segmental flexion-extension ROM with preloading. (B) CFLs (joint reaction forces) in all lumbar levels along various follower load paths (FLP (-11.38), FLP (-5), FLP (0), and FLP (5))

vior reasonably well. Optimum solutions of MFs, CFLs, joint reaction moments, and the FLP (follower load path) were feasible not only for the neutral case, as in the previous study [5], but also for the other cases of various sagittal postures. In all feasible solutions, the joint reaction moments in all levels were found to be equal to zero.

In the neutral standing posture, the solutions for the global minimum cost were found along the FLP (-11.38), while the optimum solutions of CFLs and MFs were feasible along other FLPs. The values of CFLs in all levels creating CFLs along 4 different FLPs in the neutral standing case are plotted in Figure 3(B). In the flexed postures, the optimum solutions for MFs and CFLs were feasible along any curve between FLP (-30) and FLP (15), while the solutions for the global minimum cost are along FLP (15). In the extended postures, the solutions for the global minimum function were found along FLP ($30)$, and the FLPs for the feasible solutions were found in the range from FLP (-30) to FLP (-13) for $5^{\circ}$ extension and from FLP (-30) to FLP (-25) for $10^{\circ}$ extension.

When no MFs were simulated, the FE model predictions showed that the lumbar spine buckled in flexion due to the trunk weight, as shown in Figure 4(A). In contrast, when the MFs predicted from the optimization model of the neutral posture with CFLs along FLP (-11.38) were applied, the lumbar spine was found to support the trunk weight without buckling but with significant posterior sway of the trunk $(63 \mathrm{~mm})$, as shown in Figure 4(B). Such an unstable deformation led us to investigate the spinal deformations under other MFs creating CFLs along other FLPs. Interestingly, the MFs creating CFLs along FLP (0) in Figure 4(C) resulted in a very small deformation with almost no change in the lumbar lordosis (posterior sway of the trunk less than $1.0 \mathrm{~mm}$ ) even though the predicted CFLs were greater than those in the other two cases. The predicted values of MFs and CFLs in all levels for these cases are listed in Table 1, respectively.

The MFs creating the global minimum CFLs found along FLP $(-0.65)$ for $10^{\circ}$ flexion and FLP (15) for $20^{\circ}$ and $40^{\circ}$ flexion were found to result in unstable deformations of the lumbar spine. Further analyses revealed that the MFs causing trunk sway less than $10 \mathrm{~mm}$ were predicted along FLP (-1.3), FLP (0), and FLP (2) in the lumbar spine flexed by $10^{\circ}, 20^{\circ}$ and $40^{\circ}$, respectively. MFs required for the stable deformation of the lumbar spine in the flexed postures are listed in Table 1, and the magnitudes of the CFLs created by these MFs in all lumbar levels are listed in Table 2. 
Table 1

Muscle forces in $\mathrm{N}$ required to produce stable deformation (trunk sway $<10 \mathrm{~mm}$ ) in lumbar spine in neutral and flexed postures. The CFL locations were FLP (0) for neutral, FLP (-1.3) for $10^{\circ}$ flexion, FLP (0) for $20^{\circ}$ flexion, and FLP (2.0) for $40^{\circ}$ flexion. $\mathrm{L}$ and $\mathrm{S}$ indicate long and short muscles, respectively

\begin{tabular}{|c|c|c|c|c|c|c|}
\hline & Recruited Muscles & & Neutral & ${\text { FLEX } 10^{\circ}}^{\circ}$ & FLEX $20^{\circ}$ & FLEX $40^{\circ}$ \\
\hline 1 & SerratusPI L1 Rib11 & $\mathrm{L}$ & 0.0 & 25.5 & 0.3 & 0.0 \\
\hline 2 & SerratusPI_L2_Rib12 & $\mathrm{L}$ & 0.0 & 12.9 & 0.0 & 0.0 \\
\hline 3 & LatissimusDorsi_L1_RibHum & $\mathrm{L}$ & 106.5 & 0.0 & 0.0 & 0.0 \\
\hline 4 & LatissimusDorsi_L2_RibHum & $\mathrm{L}$ & 3.5 & 0.0 & 0.0 & 0.0 \\
\hline 5 & ExternalOb_Pel_Rib10 & $\mathrm{L}$ & 7.5 & 0.0 & 0.0 & 0.0 \\
\hline 6 & ExternalOb_Pel_Rib12 & $\mathrm{L}$ & 0.0 & 0.9 & 0.0 & 0.0 \\
\hline 7 & InternalOb_Pel_Rib10 & $\mathrm{L}$ & 0.0 & 3.3 & 13.3 & 55.2 \\
\hline 8 & Longissimus_Sa_Rib11 & $\mathrm{L}$ & 0.0 & 0.0 & 0.0 & 58.3 \\
\hline 9 & Longissimus_Sa_Rib12 & $\mathrm{L}$ & 0.0 & 0.0 & 2.8 & 2.5 \\
\hline 10 & Longissimus_L3_T3 & $\mathrm{L}$ & 0.0 & 40.9 & 1.4 & 17.7 \\
\hline 11 & Longissimus_L5_T6 & $\mathrm{L}$ & 0.0 & 0.5 & 31.5 & 10.3 \\
\hline 12 & Longissimus_Sa_T11 & $\mathrm{L}$ & 0.0 & 0.0 & 0.0 & 58.3 \\
\hline 13 & Longissimus_Sa_T12 & $\mathrm{L}$ & 0.0 & 0.0 & 45.3 & 58.3 \\
\hline 14 & RecAbdominis_Pel_Rib7 & $\mathrm{L}$ & 47.3 & 0.0 & 0.0 & 0.0 \\
\hline 15 & Intertransversarii_L1_T12_Me & $\mathrm{S}$ & 0.0 & 2.7 & 45.0 & 45.0 \\
\hline 16 & Rotatores_L1_T12 & $\mathrm{S}$ & 45.0 & 0.0 & 0.0 & 0.0 \\
\hline 17 & Rotatores_L2_T12 & $\mathrm{S}$ & 45.0 & 0.0 & 0.0 & 0.0 \\
\hline 18 & Multifidus_L4_L1_F4 & $\mathrm{L}$ & 39.1 & 0.0 & 0.0 & 0.0 \\
\hline 19 & Interspinales_L2_L1 & $\mathrm{S}$ & 6.6 & 21.2 & 25.3 & 8.0 \\
\hline 20 & Intertransversarii_L2_L1_Me & $\mathrm{S}$ & 0.0 & 0.0 & 27.5 & 17.4 \\
\hline 21 & Rotatores_L2_L1 & $\mathrm{S}$ & 45.0 & 0.0 & 0.0 & 0.0 \\
\hline 22 & Rotatores_L3_L1 & $\mathrm{S}$ & 45.0 & 0.0 & 0.0 & 0.0 \\
\hline 23 & Interspinales_L3_L2 & $\mathrm{S}$ & 30.8 & 21.2 & 34.8 & 0.0 \\
\hline 24 & Intertransversarii_L3_L2_Me & $\mathrm{S}$ & 0.0 & 13.8 & 14.7 & 0.0 \\
\hline 25 & Rotatores_L3_L2 & $\mathrm{S}$ & 27.9 & 0.0 & 0.0 & 45.0 \\
\hline 26 & Longissimus_Sa_L3 & $\mathrm{L}$ & 0.0 & 22.4 & 11.7 & 0.0 \\
\hline 27 & Interspinales_L4_L3 & $\mathrm{S}$ & 32.8 & 45.0 & 16.6 & 45.0 \\
\hline 28 & Intertransversarii_L4_L3_La & $\mathrm{S}$ & 0.0 & 0.7 & 0.0 & 0.0 \\
\hline 29 & Intertransversarii_L4_L3_Me & $\mathrm{S}$ & 45.0 & 45.0 & 45.0 & 0.0 \\
\hline 30 & Rotatores_L4_L3 & $\mathrm{S}$ & 0.0 & 0.0 & 0.0 & 40.3 \\
\hline 31 & Longissimus_Sa_L4 & $\mathrm{L}$ & 91.4 & 90.4 & 104.4 & 47.6 \\
\hline 32 & Interspinales_L5_L4 & $\mathrm{S}$ & 18.3 & 12.9 & 0.0 & 0.5 \\
\hline 33 & Intertransversarii_L5_L4_Me & $\mathrm{S}$ & 45.0 & 44.1 & 0.4 & 45.0 \\
\hline 34 & Longissimus_Sa_L5 & $\mathrm{S}$ & 84.3 & 72.2 & 69.1 & 104.3 \\
\hline
\end{tabular}

In the extension posture cases, however, all 160 combinations of the MFs creating CFLs along all feasible FLPs in the extended spine were predicted to result in posterior trunk sway greater than 10 $\mathrm{mm}$. Such instability in extended posture seemed to occur because the CFL creating MFs are not feasible along FLPs close to the base spinal curve (i.e., FLP (0)) when the muscle force capacity (MFC) is $45 \mathrm{~N} / \mathrm{cm}^{2}$. 


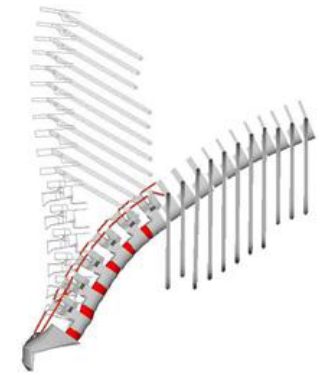

(A)

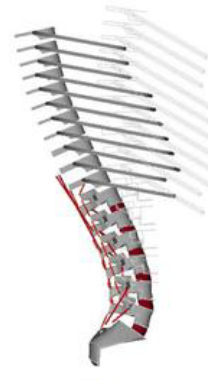

(B)

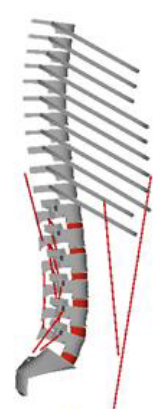

(C)

Fig. 4. Schematics of FE model predictions of spinal deformation: (A) anterior buckling of lumbar spine due to upper body weight with no contribution from spinal muscles; (B) unstable deformation with substantial posterior sway (63 mm) of trunk resulting from application of spinal MFs creating CFLs along FPL(-11.38) in addition to upper body weight; and (C) stable deformation with almost no posterior sway $(0.7 \mathrm{~mm})$ of trunk under application of spinal muscle forces creating CFLs along FLP $(0.0)$ in addition to upper body weight.

Table 2

Compressive follower loads in $\mathrm{N}$ created by muscle forces at all lumbar levels of spine in neutral and flexed postures. The CFL locations were FLP (0) for neutral, FLP (-1.3) for $10^{\circ}$ flexion, FLP (0) for $20^{\circ}$ flexion, and FLP (2.0) for $40^{\circ}$ flexion.

\begin{tabular}{lllll}
\hline & Neutral & Flexion $10^{\circ}$ & Flexion $20^{\circ}$ & Flexion $40^{\circ}$ \\
\hline Trunk-L1 & 747 & 500 & 620 & 896 \\
L1-L2 & 753 & 499 & 624 & 893 \\
L2-L3 & 697 & 511 & 623 & 893 \\
L3-L4 & 683 & 570 & 667 & 887 \\
L4-L5 & 735 & 656 & 729 & 949 \\
L5-Sa & 750 & 670 & 778 & 997 \\
\hline
\end{tabular}

\section{Discussion}

In this study, 3D optimization and FE models of the spinal system were developed, validated, and used interactively in order to investigate whether the spinal MFs creating CFLs are able to support the spinal load in various sagittal postures. Optimization solutions of MFs, joint reaction forces (CFLs), and FLP locations were feasible for all studied postures, and it was also possible to determine the MFs creating CFLs along a FLP fixed within a certain range.

The FE predictions using LS-DYNA or an explicit nonlinear code clearly demonstrated that MFs creating CFLs along FLP (0), FLP (-1.3), FLP (0), and FLP (2.0) produce stable deformation of the lumbar spine (i.e., result in trunk sway of less than $10 \mathrm{~mm}$, which could be avoided under an explicit nonlinear code) in the neutral standing and flexed postures for $10^{\circ}, 20^{\circ}$, and $40^{\circ}$ flexion, respectively, whereas the MFs creating the smallest (or optimum) CFLs resulted in unstable deformation. However, in the case of extended postures, it was not possible to find the CFL creating MFs that produced a stable deformation of the extended spine.

The validity of the FE model of the lumbar spine was confirmed by good agreement between ROMs predicted from the FE models and those measured in various studies of the lumbar spine, as presented in the results section. The CFL vectors determined from the FE predictions in response to the MFs 
creating CFLs along FLP (0) in the neutrally standing lumbar spine demonstrate an almost perfect match to those predicted from the optimization model (less than $1 \%$ difference) at all lumbar levels. Such a close match between the CFLs from the FE and optimization model predictions indicates that our optimization problems were formulated accurately without mathematical flaws. Furthermore, the magnitudes of CFLs predicted in this study were found to be comparable to those measured in previous in vivo, ex vivo, and in vitro experiments [1,24-26]. For example, the magnitude of the CFL along FLP (0) in the L3-L4 joint predicted in this study was found to be $690 \mathrm{~N}$ in the neutral posture, whereas the experimentally estimated compressive load at L2-L3 ranged from 500 to $800 \mathrm{~N}$. These validations clearly suggest that our optimization and FE models and the computational methods are reliable and that they may be able to simulate the in vivo spinal system reasonably well.

The limitation of this study in proving the hypothesis of lumbar spine stabilization by spinal muscles via the CFL mechanism was that the lumbar spine was investigated only in sagittal postures in this study. Another limitation of the current study was the lack of validation of muscle activity for stabilizing the lumbar spine in various postures such as the flexed or extended posture. The solution convergence was checked in every solution case, which suggested that spinal muscles are able to produce the follower load in a neutral posture and every flexed posture under a MFC of $45 \mathrm{~N} / \mathrm{cm}^{2}$. The follower loads for neutral postures were estimated higher than those in between 20 to 40 degrees of flexion. Finally, it is important to understand that the FE models of this study were formulated and validated to investigate the load-displacement behaviors of the lumbar spine and lumbar spinal segments. More realistic detailed simulation of the intervertebral discs, facet contacts, ligaments, and bones is possible but not performed in our models in order to reduce the computational cost. Therefore, only model predictions related to the load-displacement behavior were valid and presented in this study. More refined models are required to obtain reliable information on the stresses and strains in the spine under the physiological loads (upper body weight and CFL creating MFs) predicted in this study.

The innovations of this study can be summarized in two ways. The first innovation was the modeling of the intrinsic short muscles, which were crucial for the CFL creation by spinal muscles (Table 1) in this study. If the CFLs correspond to the physiological in vivo spinal load, as is postulated by Patwardhan et al. [4], the existence of healthy intrinsic short muscles would be one of the most critical requirements to prevent the occurrence of abnormal loads in the spine. The second innovation was the combined use of the optimization and FE analyses to investigate the effect of spinal MFs creating CFLs and affecting the stabilization of the lumbar spine.

\section{Conclusion}

In this study, FE models of the spinal system incorporating the possible actions of 232 spinal muscles were introduced, and comprehensive optimization was performed to investigate the feasibility of the follower mechanism under dynamic conditions. The results indicated that optimized spinal muscle forces demonstrate the feasibility of using CFL to stabilize the lumbar spine in vivo. This supports the hypothesis that the lumbar spine is stabilized in vivo by spinal MFs via the follower load mechanism. 


\section{Acknowledgement}

This work was supported in part by Korea Small and Medium Business Administration (No. S2023416). The authors sincerely thank ESI Korea, Co., Ltd. for their permission to use the finite element software LS-Dyna.

\section{References}

[1] A. Nachemson, Lumbar intradiscal pressure, experimental studies on post-mortem material, Acta. Orthop. Scand. Suppl. 43 (1960), 1-104.

[2] J.J. Crisco, M.M. Panjabi, I. Yamamoto and T.R. Oxland, Euler stability of the human ligamentous spine, part II: Experiment, Clin. Biomech. 7 (1992), 27-32.

[3] A.G. Patwardhan, R.M. Havey, K.P. Meade, B. Lee and B. Dunlap, Load-carrying capacity of the human cervical spine in compression is increased under a follower load, Spine (Phila Pa 1976) 25 (2000), 1548-1554.

[4] A.G. Patwardhan, R.M. Havey, K.P. Meade, B. Lee and B. Dunlap, A follower load increases the load-carrying capacity of the lumbar spine in compression, Spine (Phila Pa 1976) 24 (1999), 1003-1009.

[5] K.S. Han, A. Rohlmann, S.J. Yang, B.S. Kim and T.H. Lim, Spinal muscles can create compressive follower loads in the lumbar spine in a neutral standing posture, Med. Eng. Phys. 33 (2011), 472-478.

[6] A. Schultz, G. Andersson, R. Ortengren, K. Haderspeck and A. Nachemson, Loads on the lumbar spine, validation of a biomechanical analysis by measurements of intradiscal pressures and myoelectric signals, J. Bone. Joint. Surg. Am. 64 (1982), 713-720.

[7] A.G. Patwardhan, K.P. Meade and B. Lee, A frontal plane model of the lumbar spine subjected to a follower load: Implications for the role of muscles, J. Biomech. Eng. 123 (2001), 212-217.

[8] H.J. Wilke, A. Rohlmann, S. Neller, F. Graichen, L. Claes and G. Bergmann, ISSLS prize winner: A novel approach to determine trunk muscle forces during flexion and extension: A comparison of data from an in vitro experiment and in vivo measurements, Spine (Phila Pa 1976) 28 (2003), 2585-2593.

[9] M. El-Rich, A. Shirazi-Adl and N. Arjmand, Muscle activity, internal loads, and stability of the human spine in standing postures: Combined model and in vivo studies, Spine (Phila Pa 1976) 29 (2004), 2633-2642.

[10] I.A. Stokes and M. Gardner-Morse, Lumbar spine maximum efforts and muscle recruitment patterns predicted by a model with multijoint muscles and joints with stiffness, J. Biomech. 28 (1995), 173-186.

[11] S.H. Zhou, I.D. McCarthy, A.H. McGregor, R.R. Coombs and S.P. Hughes, Geometrical dimensions of the lower lumbar vertebrae--analysis of data from digitised CT images, Eur. Spine. J. 9 (2000), 242-248.

[12] R.M. Lin, I.M. Jou and C.Y. Yu, Lumbar lordosis: Normal adults, J. Formos. Med. Assoc. 91 (1992), 329-333.

[13] T. Brown, Some mechanical tests on the lumbosacral spine with particular reference to the intervertebral discs, J. Bone Joint Surg. Am. 39 (1957), 1135-1164.

[14] N. Bogduk, J.E. Macintosh and M.J. Pearcy, A universal model of the lumbar back muscles in the upright position, Spine (Phila Pa 1976) 17 (1992), 897-913.

[15] N. Bogduk, M.J. Pearcy and G. Hadfield, Anatomy and biomechanics of psoas major, Clin. Biomech. 7 (1992), 109119.

[16] J.E. Macintosh, J.E. and N. Bogduk, The morphology of the human lumbar multifidus, Clin. Biomech. 1 (1986), 196204.

[17] S.M. McGill, N. Patt and R.W. Norman, Measurement of the trunk musculature of active males using CT scan radiography: Implications for force and moment generating capacity about the L4/L5 joint, J. Biomech. 21 (1988), 329-341.

[18] E. Charriere, F. Sirey and P.K. Zysset, A finite element model of the L5-S1 functional spinal unit: Development and comparison with biomechanical tests in vitro, Comput. Methods Biomech. Biomed. Engin. 6 (2003), 249-261.

[19] M.M. Panjabi, T.R. Oxland, R.M. Lin and T.W. McGowen, Thoracolumbar burst fracture, a biomechanical investigation of its multidirectional flexibility, Spine (Phila Pa 1976) 19 (1994), 578-585.

[20] P. Vena, G. Franzoso, D. Gastaldi, R. Contro and V. Dallolio, A finite element model of the L4-L5 spinal motion segment: Biomechanical compatibility of an interspinous device, Comput. Methods Biomech. Biomed. Engin. 8 (2005), $7-$ 16.

[21] J. Janevic, J.A. Ashton-Miller and A.B. Schultz, Large compressive preloads decrease lumbar motion segment flexibility, J. Orthop. Res. 9 (1991), 228-236. 
[22] M.G. Gardner-Morse and I.A. Stokes, Structural behavior of human lumbar spinal motion segments, J. Biomech. 37 (2004), 205-212.

[23] S.M. Renner, R.N. Natarajan, A.G. Patwardhan, R.M. Havey, L.I. Voronov, B.Y. Guo, G.B. Andersson and H.S. An, Novel model to analyze the effect of a large compressive follower pre-load on range of motions in a lumbar spine, J. Biomech. 40 (2007), 1326-1332.

[24] C.R. Dennison, P.M. Wild, P.W. Byrnes, A. Saari, E. Itshayek and D.C. Wilson, Ex vivo measurement of lumbar intervertebral disc pressure using fibre-Bragg gratings, J. Biomech. 41 (2008), 221-225.

[25] D.S. McNally and M.A. Adams, Internal intervertebral disc mechanics as revealed by stress profilometry, Spine (Phila Pa 1976) 17 (1992), 66-73.

[26] A. Rohlmann, T. Zander, M. Rao and G. Bergmann, Applying a follower load delivers realistic results for simulating standing, J. Biomech. 42 (2009), 1520-1526. 